\title{
A High-speed Train Timetable Rescheduling Approach in case of Disturbances
}

\author{
Xinyu Gao ${ }^{1, a}$ \\ ${ }^{1}$ School of Electronic and Information Engineering, Beijing Jiaotong University, China
}

\begin{abstract}
This paper from a macroscopic viewpoint develops a train timetable rescheduling approach on a single high-speed railway line under disturbances, i.e. inevitable train delays in the duration of the train operation. A mixedinteger linear programming model is formulated to minimize the arrival delay and the departure delay altogether. The commercial optimization software CPLEX is adopted in an effort to seek the optimal solution in an acceptably short time required in the real-time rescheduling process. The proposed approach is further tested on a real-world case study and the numerical results show that compared with the results obtain by the traditional genetic algorithm, using CPLEX to solve the model can yield better solutions and consume the desired computation time, thereby demonstrating its effectiveness and efficiency.
\end{abstract}

\section{Introduction}

High-speed railway has developed greatly in recent decades. In China, the large-scale network of railway has been put into use, which poses enormous challenges concerning the safe and efficient operation of high-speed trains when unexpected disturbances occur. In such disrupted cases, the operation of high-speed trains deviates from the planned train diagram, causing train delays which further leads to passenger dissatisfaction as well as economic losses, all of which therefore attach paramount importance to timely timetable rescheduling approaches. The high-speed railway planning process is shown in Figure 1, where the train real time rescheduling, especially the train timetable rescheduling in the operational level has been paid enormous attention by researchers at home and abroad.

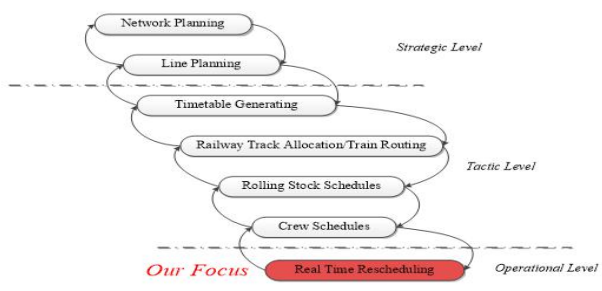

Figure 1. The high-speed railway hierarchical planning process

a Corresponding author: 1039789301@qq.com
In the recent study, Higgins et al [1] developed a model for the online train timetable rescheduling and introduced a branch and bound algorithm to minimize the total delay of all trains as well as the train operating cost, which acts as a decision-making tool for train dispatchers to reschedule trains in real time to an optimal outcome. Corman et al [2] proposed an optimization framework by using alternative graphs to model train operation at the microscopic level that considers the role of the block sections and signals. In an attempt to collectively minimize the energy consumption and passenger waiting time, Meng et al [3] formulated and solved a robust single-track train dispatching problem under stochastic segment running times and capacity loss duration. Dong et al [4] integrated artificial systems, computational experiments, and parallel execution, also known as ACP approach, to model complex urban rail transport systems when confronted with emergencies. D'Ariano et al [5] proposed a branch and bound algorithm for solving the rescheduling problem in real time. D'Ariano et al [6] further implemented the alternative graph model in the real-time train rescheduling to support dispatchers to resolve conflicts. Espinosa-Aranda et al [7] introduced a binary integer linear programming to minimize total passenger delays and developed a heuristic method to solve the model. Yang et al [8] developed a novel fuzzy programming model for rescheduling trains based on the fuzzy variable representation of incident duration. 
Cacchiani et al [9] presented an overview of the train rescheduling models and algorithms for the real-time adjustment under the disturbances and disruptions. Binder et al [10] integrated three objectives to generate a disposition timetable in the event of disruptions: the passenger satisfaction, the operational costs and the deviation from the original timetable.

In many countries, such as in China, the high-speed train adjustment is mainly dependent upon human operations. In practice, when disturbances occur, dispatchers are expected to act based on some existing contingency plans or adjust the train timetable manually. However, dispatchers tend to find it difficult to opt for the best contingency plan, and adjusting the timetable manually is time-consuming. What is more, when manually adjusting the timetable, the results are highly dependent upon the skills and experience of the dispatcher, which cannot guarantee the optimal quality of the results. Therefore, in this paper, a mix-integer linear programming mathematical optimization model is constructed and the CPLEX solver is used, which can serve as a tool to help dispatchers with the decisionmaking process.

\section{Model formulation}

In this section, the detailed mathematical formulation of the rescheduling optimization model is presented in case of disturbances. In the proposed model, the timetable is considered at a macroscopic level, which means that it is represented by the arrival and departure times of each train at each station and detailed information about signaling systems is neglected.

No-early constraints: These following constraints ensure that rescheduled departure and arrival times of all trains cannot be earlier than their scheduled counterparts since it would cause trouble for the travelling of passengers who book the train tickets in advance. Therefore the constraints are presented as:

$$
\begin{aligned}
& t_{i s}^{d}-o_{i s}^{d} \geq 0 \quad \forall i \in I, \forall s \in\{1,2, \ldots,|S|-1\} \\
& t_{i s}^{a}-o_{i s}^{a} \geq 0 \quad \forall i \in I, \forall s \in\{2,3, \ldots,|S|\}
\end{aligned}
$$

Where $o_{i s}^{d}, o_{i s}^{a}$ are scheduled departure times and arrival times of train $i$ in the intermediate station $s$, and $t_{i s}^{d}, t_{i s}^{a}$ are their rescheduled counterparts. $I$ is the set of trains where $i, j$ are indexes of trains, and $S$ is the set of stations where $s, h$ are indexes of stations. $|I|,|S|$ are total numbers of trains and stations respectively.

Train delay constraints: For trains that run properly as scheduled, the arrival and departure delays are formed respectively as follows:

$$
\begin{aligned}
& d_{i s}^{d} \geq t_{i s}^{d}-o_{i s}^{d} \forall i \in I, \forall s \in\{1,2, \ldots,|S|-1\} \\
& d_{i s}^{a} \geq t_{i s}^{a}-o_{i s}^{a} \forall i \in I, \forall s \in\{2,3, \ldots,|S|\}
\end{aligned}
$$

where $d_{i s}^{d}, d_{i s}^{a}$ suggest the departure delay and the arrival delay of train $i$ at station $s$. It should be noted that trains departing at the initial station should not be delayed and that trains arriving at the final station are without delays as they are no longer used for transport.

Train running constraints: The time that trains run between stations can be calculated as below, and it is worth noting that train cannot surpass its maximum speed.

$$
\begin{aligned}
& t_{i, s-1}^{d} \leq t_{i s}^{d} \forall i \in I, \forall s \in\{1,2, \ldots,|S|-1\} \\
& t_{i, s-1}^{a} \leq t_{i s}^{a} \forall i \in I, \forall s \in\{2,3, \ldots,|S|\} \\
& t_{r} \geq t_{i, s+1}^{a}-t_{i s}^{d} \forall i \in I, \forall s \in\{1,2, \ldots,|S|-1\} \\
& t_{r} \geq l_{s} / V \quad \forall i \in I, \forall s \in\{1,2, \ldots,|S|-1\}
\end{aligned}
$$

where $t_{r}$ represents the running time between intermediate stations, and $l_{s}$ indicates the distance between stations.

Dwelling time constraints:If a train is pre-scheduled to stop at a specific station, some time should be given for this train to carry out necessary operations, for example loading and unloading passengers as well as changing crew members, etc. It is obvious that if the arrival and departure times of each train $i$ at each station $s$, the actual dwelling time $p_{i s}$ for train $i$ at station $s$ can be calculated by the difference of the rescheduled arrival and departure times, which is $p_{i s}=t_{i s}^{d}-t_{i s}^{a}$. Therefore, the dwelling time should be no less than a pre-determined minimum time $t_{d w e}$. The dwelling time constraint at intermediate stations are here as follows:

$$
t_{i s}^{d}-t_{i s}^{a} \geq x_{i s} \cdot t_{d w e} \forall i \in I, \forall s \in\{1,2, \ldots,|S|-1\}
$$

Headway constraints: In order to guarantee the safe operations on the railway line, headway constraints need to be formulated to keep a safe distance between the adjacent trains, including the arrival and the departure headway at stations. Specifically, if a train arrives at/departs from a station, a time period should be left to the preparation for the next train's arrival/departure. In other words, this time period is the time distance of two trains for their arrival or departure operations. A binary variable $y_{i j s}$ is here introduced, suggesting if $y_{i j s}=1$ it means train $i$ departs before train $j$ at station $s$, and $y_{i j s}=0$ means the other way round. This binary variable ensures that the overtaking operation can be achieved within stations if necessary. The following constraints therefore indicate that the departure and arrival headway at stations should be assured between adjacent trains that are scheduled to stop. All can be described as follows:

$$
\begin{aligned}
& t_{j s}^{d}-t_{i s}^{d} \geq t_{d e p}+M \cdot\left(y_{i j s}-1\right) \quad \forall i, j \in I, \forall s \in\{1,2, . .|S|-1\} \\
& t_{j s}^{a}-t_{i s}^{a} \geq t_{a r r}+M \cdot\left(y_{i j s}-1\right) \quad \forall i, j \in I, \forall s \in\{2,3, . .|S| S\} \\
& t_{i s}^{d} \leq t_{j s}^{d}+M \cdot\left(1-y_{i j s}\right) \quad \forall i, j \in I, \forall s \in\{1,2, . .|S|-1\} \\
& t_{i s}^{d} \geq t_{j s}^{d}-M \cdot y_{i j s} \quad \forall i, j \in I, \forall s \in\{1,2, . . .|S|-1\} \\
& y_{i j s}+y_{j i s}=1 \quad \forall i, j \in I, i \neq j, \forall s \in\{1,2, . .|S|-1\}
\end{aligned}
$$

In the meantime, the departure and arrival headway, which is the headway between the departure time of the former train and the arrival time of the latter train, should also be satisfied as follows:

$$
o_{i s}^{d}-o_{i s}^{a} \leq t_{i s}^{d}-t_{i s}^{a} \quad \forall i \in I, \forall s \in S
$$


Capacity constraints: To ensure that the rescheduled timetable is feasible in real cases, station capacities represented by tracks should better be taken into consideration in the duration of the rescheduling process. It is common knowledge that trains arriving in a station to stop or passing the station should be assigned to a station track. A station track can be occupied by only one train at one time. Note that like in the most literature it is assumed that the origin and destination stations where the train services start and end have the enough capacity to store the starting and ending trains. The capacity constraints are therefore constructed, and it is worth mentioning that the first two constraints represent that if these two constraints are true, they together mean that a train is at the station stopping or passing, which requires an empty track.

$$
\begin{aligned}
& \left(t-t_{i s}^{a}\right) \leq j_{i s t}^{a} \cdot M \quad \forall t \in T T, \forall i \in I, \forall s \in S \\
& \left(t_{i s}^{d}-t\right) \leq j_{i s t}^{d} \cdot M \quad \forall t \in T T, \forall i \in I, \forall s \in S \\
& j_{i s t}^{p} \geq\left(j_{i s t}^{a}+j_{i s t}^{d}-2\right) \cdot M+1 \quad \forall t \in T T, \forall i \in I, \forall s \in S \\
& \sum j_{i s t}^{p} \leq C_{s} \quad \forall t \in T T, \forall i \in I, \forall s \in S
\end{aligned}
$$

where the binary variables $j_{i s t}^{d}, j_{i s t}^{a}, j_{i s t}^{p}$ are constructed variables for the formulation of the capacity constraints, and $\mathrm{Cap}_{s}$ represents the actual number of available tracks. TT indicates the whole time horizon of the rescheduling process.

Objective function: The objective function is constructed as:

$$
\min Z=\sum_{i=1}^{|I|} \sum_{s=1}^{|S|}\left(\alpha_{1} \cdot d_{i s}^{d}+\alpha_{2} \cdot d_{i s}^{a}\right)
$$

where in the function the weighted-sum optimization method is used, in which $\alpha_{1}, \alpha_{2}$ are weight coefficients, to aim at minimizing the departure delay and the arrival delays of all trains, so as to restore the normal railway operation of trains as soon as possible and diminish the dissatisfaction of passengers in cases of disturbances.

\section{Case study}

In order to test and verify the effectiveness and efficiency of the proposed approach, in this section, a real-world case study is carried out based upon the data of Shanghai Hongqiao station to Nanjing South station in the upstream direction. The simulation is coded in Matlab 2016a and performed on an Intel(R) Core(TM) i7-8550U CPU@1.80 GHz 2.00 GHz running with a 64-bit windows operating system and $8 \mathrm{G}$ RAM. The commercial optimization software CPLEX 12.6 is adopted to solve the proposed the mix-integer linear programming model.

High-speed railway lines generally have double tracks with one track for each direction, and the upstream direction is considered on the assumption that sidings for upstream trains can only be used by upstream trains and vice versa. The number of tracks are set in accordance with half of the reality because only the upstream direction is used. As regards parameters, $t_{d w e}$ is set as 2 $\min , t_{d e p}, t_{\text {arr }}$ are $3 \mathrm{~min}, t_{q}, t_{t}$ are $0.5 \mathrm{~min}, V$ is $300 \mathrm{~km} / \mathrm{h}$, $M$ is $1000000, l_{s}$ is set according to the data of reality, and $\alpha_{1}, \alpha_{2}$ are set as 5 in the following case study which can be altered to study the influence of the coefficients.

As is illustrated in Figure 2, the real-world planned timetable---from Shanghai Hongqiao station to Nanjing South station within the time horizon from 6:00 a.m. to 11:00 a.m. covering 8 stations and 10 high-speed trains--acts as the benchmark for the rescheduling stage with blue lines.

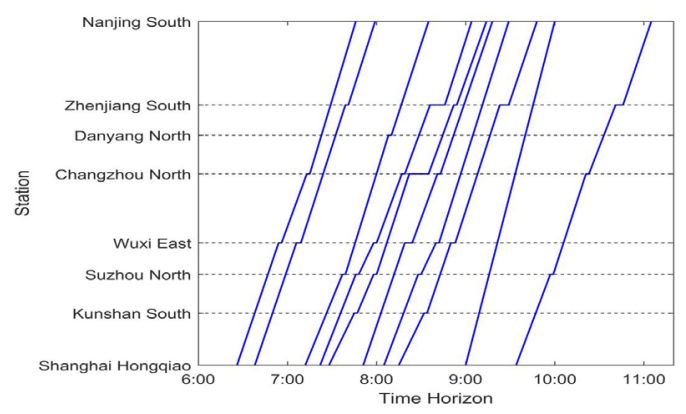

Figure 2. Planned high-speed train diagram.

Two typical disturbance scenario are considered: the fourth train departs from the fourth station with $8 \mathrm{~min}$ delays, and the fourth train arrives at the fourth station with 8 min delays.

As for the first departure delay scenario, Figure 3 and Figure 4 show the results obtained by the traditional standard genetic algorithm and CPLEX respectively. It can be seen that both can provide non-conflicting feasible solutions with red lines. However, the total delay calculated by genetic algorithm is $104.75 \mathrm{~min}$, and by CPLEX is $36.2 \mathrm{~min}$, showing that CPLEX can find better solutions by reducing delays by approximately $65 \%$. At the same time, the Gap of CPLEX is 0 , meaning that the solution by CPLEX solver is the optimal one. The computation time is 41.6778 s and 98.3569 s respectively, which can also suggest that using CPLEX solver can get the solutions faster so as to fulfil the real-time performance. The delay reduced and the time shortened therefore demonstrate the effectiveness and efficiency of the proposed approach.

With regard to the second arrival delay scenario, Figure 5 and Figure 6 show the results acquired by the traditional standard genetic algorithm and CPLEX respectively. The total delays calculated by genetic algorithm is $134.4420 \mathrm{~min}$, while by CPLEX is 70.9014 min with the Gap of CPLEX also 0, which indicates that CPLEX can achieve better results by reducing train delays by about $47 \%$. The computation time is $24.3677 \mathrm{~s}$ and $95.3468 \mathrm{~s}$ respectively, showing that CPLEX can better achieve the real-time performance as well. All therefore demonstrate the effectiveness and efficiency of the proposed approach. 


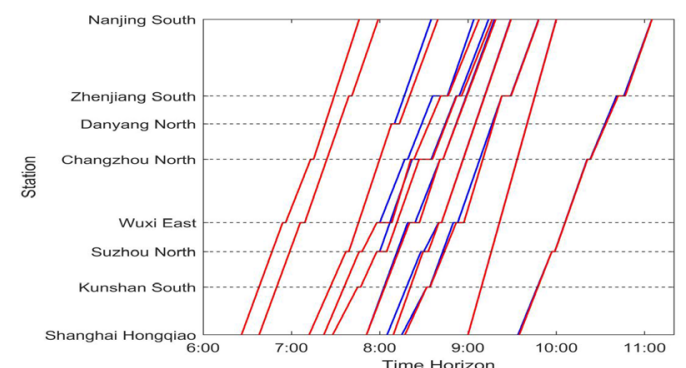

Figure 3. Rescheduled train diagram obtained by the genetic algorithm for departure delay

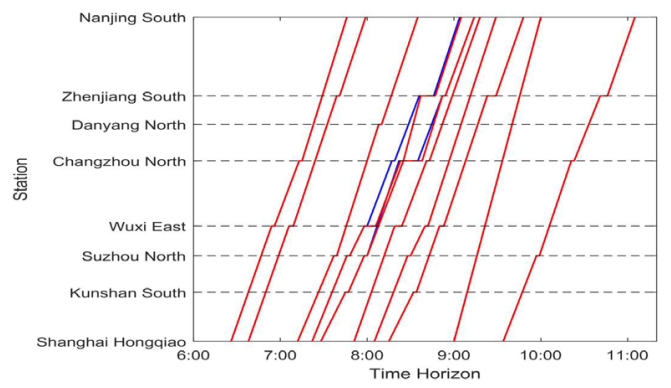

Figure 4. Rescheduled train diagram obtained by CPLEX for departure delay

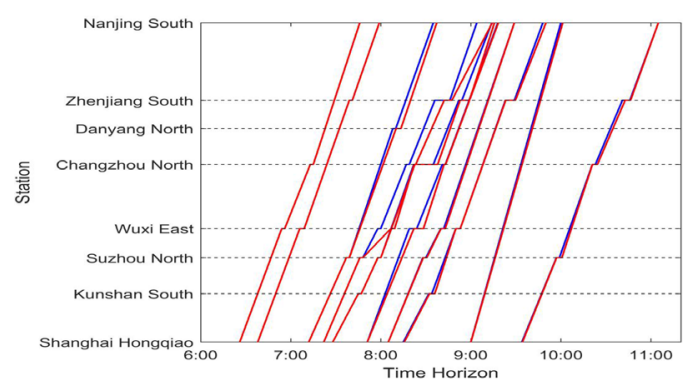

Figure 5. Rescheduled train diagram obtained by the genetic algorithm for arrival delay

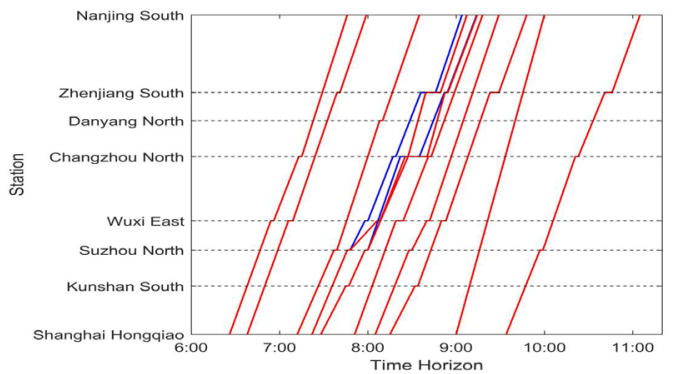

Figure 6. Rescheduled train diagram obtained by CPLEX for arrival delay

\section{Conclusion}

In this paper, the issue of the high-speed train timetable rescheduling process under disturbances was explored. Specifically, to minimize the departure and arrival delay of all trains, a mixed-integer linear programming model was formulated. The commercial CPLEX solver was used to solve the proposed model. A case study was presented to verify the effectiveness and efficiency of the proposed approach. The simulation results showed that compared with the results attained by the genetic algorithm, CPLEX solver can obtain better solutions in terms of train delays and the computation time. For future research, high-speed train rescheduling models which integrate train timetable, rolling stock and crew staff should be studied, and novel intelligent algorithms should be explored in order to better the rescheduling process.

\section{References}

1. A. Higgins, E. Kozan, L. Ferreira, Transportation Research Part B: Methodological, 30,147, (1996)

2. F. Corman, A. DAriano, I. A. Hansen, and D. Pacciarelli, Journal of Rail Transport Planning \& Management, 1, 14, (2011)

3. L. Meng and X. Zhou, Transportation Research Part B: Methodological, 45, 1080, (2011)

4. H. Dong, B. Ning, Y. Chen, X. Sun, D. Wen, Y. Hu, and R. Ouyang, IEEE Transactions on Intelligent Transportation Systems, 14, 627, (2013)

5. A. Dariano, D. Pacciarelli, and M. Pranzo, European Journal of Operational Research, 183, 643, (2007)

6. A. D' Ariano, F. Corman, D. Pacciarelli, and M. Pranzo, Transportation science, 42, 405 - 419, (2008)

7. J. L. Espinosa-Aranda and R. Garcia-Ródenas, Journal of Rail Transport Planning \& Management, 3, 1, (2013)

8. L. Yang, X. Zhou, and Z. Gao, Omega, 48, 75, (2014)

9. V. Cacchiani, D. Huisman, M. Kidd, L. Kroon, P. Toth, L. Veelenturf, and J. Wagenaar, Transportation Research Part B: Methodological, 63, $15,(2014)$

10. S. Binder, Y. Maknoon, and M. Bierlaire, Transportation Research Part C: E-merging Technologies, 78, 78, (2017) 